\title{
Optimization of tumor xenograft dissociation for the profiling of cell surface markers and nutrient transporters
}

\author{
Vincent Petit ${ }^{1,2,7,8}$, Gérald Massonnet ${ }^{3,7}$, Zofia Maciorowski ${ }^{4}$, Jawida Touhami ${ }^{1,2}$, Aurélie Thuleau ${ }^{3}$, Fariba Némati ${ }^{3}$, \\ Julie Laval ${ }^{1,2}$, Sophie Château-Joubert ${ }^{5}$, Jean-Luc Servely ${ }^{5}$, David Vallerand ${ }^{3}$, Jean-Jacques Fontaine ${ }^{5}$, \\ Naomi Taylor ${ }^{1,2}$, Jean-Luc Battini ${ }^{1,2}$, Marc Sitbon ${ }^{1,2}$ and Didier Decaudin ${ }^{3,6}$
}

Metabolic adaptations and changes in the expression of nutrient transporters are known to accompany tumorigenic processes. Nevertheless, in the context of solid tumors, studies of metabolism are hindered by a paucity of tools allowing the identification of cell surface transporters on individual cells. Here, we developed a method for the dissociation of human breast cancer tumor xenografts combined with quantification of cell surface markers, including metabolite transporters. The expression profiles of four relevant nutrient transporters for cancer cells' metabolism, Glut1, ASCT2, PiT1 and PiT2 (participating to glucose, glutamine and inorganic phosphate, respectively), as detected by new retroviral envelope glycoprotein-derived ligands, were distinctive of each tumor, unveiling underlying differences in metabolic pathways. Our tumor dissociation procedure and nutrient transporter profiling technology provides opportunities for future basic research, clinical diagnosis, prognosis and evaluation of therapeutic responses, as well as for drug discovery and development.

Laboratory Investigation (2013) 93, 611-621; doi:10.1038/labinvest.2013.44; published online 4 March 2013

KEYWORDS: breast cancer; cell surface transportome; flow cytometry analysis; live cells; metabolite transporters; tumor dissociation; xenografts

Markers of tumor cell metabolism are of valuable importance for basic and clinical cancer research. The identification of metabolic alterations that accompany cancer processes is becoming critical for refining the clinical evaluation and treatment of patients, ${ }^{1,2}$ as well as for further drug discovery and development. While metabolomics relies mainly on the quantification of anabolic and catabolic end products, ${ }^{3}$ the bidirectional fluxes of nutrients and metabolites, as well as the efflux of toxins and pharmaceutical compounds, are ensured by nutrient transporters. ${ }^{4}$ However, the monitoring of metabolite and nutrient transporters at the cell surface has been hampered by the paucity, if not total lack, of exofacial antibodies to such transporters. ${ }^{5,6}$ Cell entry of gamma- and deltaretroviruses occurs via recognition of membrane metabolite transporters by retroviral envelope glycoproteins (Env). ${ }^{7,8}$ Viral entry occurs following the specific binding of the amino-terminal receptor binding domain (RBD) of an individual Env with its cognate receptor. We designed RBD probes, derived from gamma- and deltaretroviral Env, as specific ligands that bind to these cell surface transporters. Binding of defined ligands allows the quantification of distinct sets of metabolite transporters at the cell surface. ${ }^{9-13}$ Furthermore, while cell surface labeling is readily achieved on cultured cell lines and circulating hematopoietic cells, singlecell labeling of solid tumors has remained problematic. Indeed, such analyses require dissociation methods that yield

\footnotetext{
'Institut de Génétique Moléculaire de Montpellier (IGMM), CNRS, Montpellier, France; ${ }^{2}$ Université Montpellier 2; Université Montpellier 1, Montpellier, France; ${ }^{3}$ Laboratory of Preclinical Investigation, Translational Research Department, Institut Curie, Paris, France; ${ }^{4}$ Flow Cytometry Core Facility, Institut Curie, Paris, France;

${ }^{5}$ Université Paris-Est, Ecole Nationale Vétérinaire d'Alfort, Unité d'Anatomie Pathologique, Maisons-Alfort, France and ${ }^{6}$ Department of Medical Oncology, Institut Curie, Paris, France

Correspondence: Dr M Sitbon, PhD, Institut de Génétique Moléculaire de Montpellier, CNRS, 1919 Route de Mende, 34293 Montpellier cedex 05, France.

E-mail: marc.sitbon@igmm.cnrs.fr or Dr D Decaudin, MD, PhD, Service d'Hématologie, Institut Curie, 26 rue d'Ulm, 75248 Paris cedex 05, France.

E-mail: didier.decaudin@curie.net

${ }^{7}$ The first two authors contributed equally to this work.

${ }^{8}$ Current address: METAFORA Biosystems, Cap Alpha, Clapiers, France

Received 21 June 2012; revised 2 February 2013; accepted 2 February 2013
} 
sufficient numbers of viable cells while maintaining the initial cell composition of the tumor. Moreover, it is important that the dissociation does not disrupt the expression and/or detection of surface antigens such as membrane transporters. Thus, the development of suitable dissociation methods that reflect tumor heterogeneity while allowing the identification of the different cell types present within the tumor, including stem cells, inflammatory cells, stroma as well as cells from the peritumoral microenvironment, are critical.

Clinical biopsies are generally unsuitable for the development of such dissociation and staining protocols, mainly due to their limited availability. Notably though, human tumors grafted and passaged in mice have been shown to maintain consistently the characteristics of the primary tumor, thus providing precious preclinical models for assay development before clinical use. ${ }^{14-16}$

The technical challenges encountered in multiparametric analyses of single viable cells from solid tissues are generally unaffected by the origin of the tissue. However, total cell recovery is critical for obtaining sufficient material for experimentation and for assessing the phenotypes of sparse cell subtypes. ${ }^{17}$ As viability and integrity are major concerns, the level of apoptosis and necrosis of the dissociated cells must be carefully assessed, especially when cells are to be further used for ex vivo culture, injection into animals or molecular analyses. Poor or selective tissue dissociation can alter the composition of cell subpopulations, thereby biasing analyses and inferred results.

Single viable cell recovery protocols include two main steps: tissue dissociation and cell purification. Tissue dissociation involves mechanical dissociation followed by enzymatic digestion, both of which are detrimental to cells. Mechanical disaggregation increases the accessibility of the tissue surface to enzymes. Enzyme cocktails must be carefully chosen and tailored to the tissue, particularly for epithelial carcinomas where the epithelial junctions (zonula occludens) are much more difficult to disrupt than in contiguous mesenchymal or stromal tissues. In terms of viability, trypsin is the most effective enzyme for cell dissociation, but short incubation times result in poor total cell recovery, and some antigens of interest are sensitive to tryptic activity. ${ }^{18}$ Hyaluronidase, collagenase and dispase, each with distinct digestive properties, listed here from the most to the least potent, are also commonly used in customized cocktails. Once a single-cell suspension has been obtained, cells of interest must be enriched, and red cells, necrotic cells and debris eliminated. Two common methods for eliminating red cells are ammonium chloride red cell lysis buffers, which can be toxic for nucleated cells, ${ }^{19}$ and Ficoll gradients, which, while eliminating dead cell and debris, can also eliminate some polyploid cells. ${ }^{20,21}$

In this study, we optimized a three-step procedure for maximum recovery of viable cells from primary human breast cancer xenografts in mice, using a non-enzymatic dissociation buffer (NEDB), followed by an enzyme cocktail of collagenase III and deoxyribonuclease type I (DNase I) and purification on dual-density Ficoll gradients. The consistency and reliability of this dissociation method was first assessed by comparing the recovery of CD44 + cells in tumor suspensions with their incidence in tissue sections, and an assessment of the mean CD44 expression level in dissociated cells as compared with sections.

This protocol was then used to monitor the CD44, CD24 and CD326 cell surface markers, as well as the expression profiles of four major membrane nutrient transporters described as key players of cancer cell biology: the glucose transporter Glut1, the neutral amino-acid transporter ASCT2 and two inorganic phosphate transporters PiT1 and PiT2. Cell surface expression of these transporters was detected using a series of specific RBD, and monitored by flow cytometry. Remarkably, the relative level of each nutrient transporter was found to be reproducible for xenografts that derived from the same tumor but differed between tumors. Indeed, metabolic transporter expression profiles were distinctive of each human breast cancer model and could be used to identify a specific tumor. Therefore, a validated protocol for tumor dissociation combined with a quantifiable phenotyping of cell surface metabolite transporters allows the elaboration of a distinctive metabolic portrait of each tumor.

\section{MATERIALS AND METHODS Human Tumor Xenografts in Mice}

Human breast cancer specimens were obtained following informed consent from patients undergoing surgery. Fresh tumor fragments were grafted into the interscapular fat pad of 8- to 12-week-old female Swiss nude mice (Harlan Laboratories, L'Arbresle, France) under avertin anesthesia. Mice were maintained in pathogen-free animal housing (Institut Curie, Paris, France) and received estrogen $(17 \mathrm{mg} / \mathrm{ml})$ diluted in drinking water. Xenografts appeared at the graft site 2-8 months after initial transplantation. They were subsequently transplanted into secondary recipients and were considered to be established as of the third in vivo passage. ${ }^{22}$ All experiments were performed in accordance with the UKCCCR animal ethics guidelines. ${ }^{23}$ Well-established tumor models with a minimum tumor size of $600 \mathrm{~mm}^{3}$ were used in this study. Main characteristics of xenografts used in this study are presented in Supplementary Table 1.

\section{Tissue Dissociation}

To maximize cell yield and viability, different combinations of enzymes, including collagenase III $(200 \mathrm{U} / \mathrm{ml}$; Sigma-Aldrich, St Louis, MO, USA), DNase I (200 U/ml; Sigma-Aldrich) and trypsin $(5 \mathrm{mg} / \mathrm{ml}$; Invitrogen, Madison, WI, USA), and an NEDB (Invitrogen) were tested.

Briefly, mice were killed, and tumors were removed into cold culture media and processed immediately. Surrounding mouse breast tissue and fat were removed. The tumors were minced into 2-4 $\mathrm{mm}$ fragments, which were then incubated with the appropriate dissociation solutions, either NEDB or 
enzymes, for $30 \mathrm{~min}$ at $37^{\circ} \mathrm{C}$. The tumor fragments were mixed up and down every $10 \mathrm{~min}$ using a $1000 \mu \mathrm{l}$ micropipette with a tip cut to a diameter adapted to tissue fragment size. After each incubation period, the fragments were filtered through a $40 \mu \mathrm{m}$ nylon mesh cell strainer (BD Biosciences, San Diego, CA, USA). The released cells were centrifuged at 1200 r.p.m. for 2 min and stored in cold $\mathrm{CO}_{2}$-independent medium with $30 \%$ FCS at $4{ }^{\circ} \mathrm{C}$. Fresh dissociation solution was added to the remaining tissue fragments for $30 \mathrm{~min}$. Dissociation was stopped when no additional cells were released. The fragments were pushed through a sieve and all cells from all incubation periods were pooled and counted.

The dissociation protocols tested are summarized in Figure 1: Protocol E (Enzymes) consisted of three consecutive incubations with collagenase III and DNase I. Protocol Try + E (Trypsin plus Enzymes) consisted of three consecutive incubations with collagenase III and DNase I supplemented with trypsin. Protocol NEDB + E (NEDB plus Enzymes) consisted of two consecutive incubations with NEDB, followed by one enzymatic digestion with collagenase III and DNase I. Protocol NEDB + E + TRY (NEDB plus Enzymes plus Trypsin) was the same as protocol NEDB $+\mathrm{E}$, with trypsin added to the collagenase III/ DNase I cocktail.

The dissociated cells were layered onto a double Ficoll gradient (Histopaque; Sigma-Aldrich; densities 1.077 and
1.119) as described for leukocyte separation and spun at $700 \mathrm{~g}$ for $30 \mathrm{~min}$ at room temperature. Cells removed from both interfaces were pooled and washed two times in $\mathrm{CO}_{2^{-}}$ independent medium and stored at $4{ }^{\circ} \mathrm{C}$. Overnight storage did not significantly alter cell viability.

\section{Cell Viability and Culture}

Cell count and viability were assessed immediately after dissociation by Trypan blue exclusion on a hemocytometer. For the ex vivo viability culture assay, 40000 cells were plated into flat bottom 96-well plates in $100 \mu$ l of high glucose DMEM with $10 \%$ FCS and glutamine, sodium pyruvate, penicillin and streptomycin at $37^{\circ} \mathrm{C}$. The viability of cultured dissociated cells over time was assessed with WST-1, an assay based on the cleavage of a tetrazolium salt by respiratory mitochondrial enzymes (Roche, Indianapolis, IN, USA). A measure of $10 \mu \mathrm{l}$ of WST-1 was added to six replicate wells at different time points. The mean optical density at $450 \mathrm{~nm}$ was plotted against time. At the final time point, cells were trypsinized and stained for mouse versus human markers to determine whether viable cells originated from the human tumor or mouse stroma.

Cultured dissociated cells were treated ex vivo with anticancer drugs. Dissociated cells (40000 per well) were aliquoted into flat bottom 96-well microplates. After 3 days, the medium was changed and docetaxel (Aventis Pharma, a

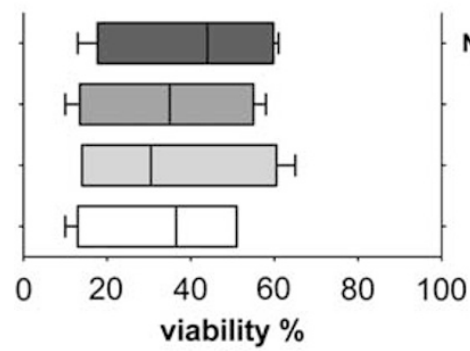

dissociation protocol efficiency

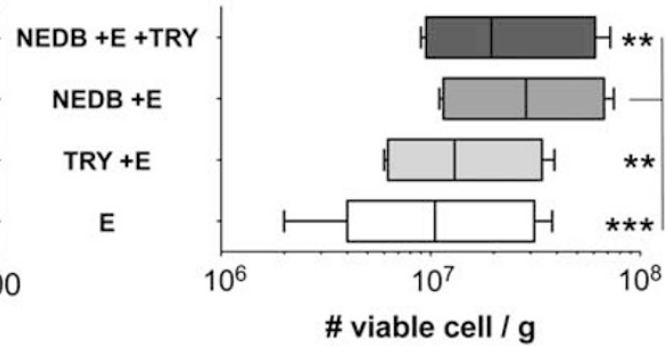

ficoll efficiency

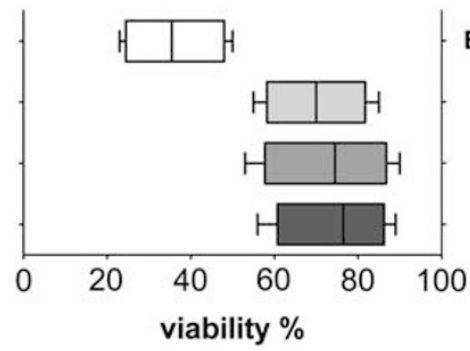

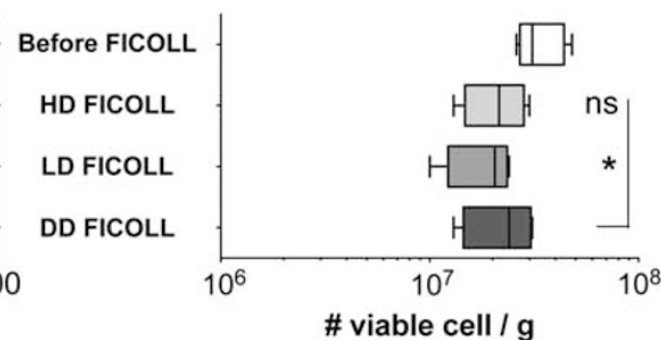

Figure 1 Yields and viability of tumor cells after different tissue dissociation and purification protocols. (a) Recovery of viable cells was determined on a representative xenograft of the indicated breast cancer models. Viability was assessed by Trypan blue exclusion after trypsin (TRY) and other enzyme (E)-based or non-enzymatic dissociation (NEDB) buffers, alone or in different combinations. Results are expressed as viable cells per gram of tumor (\% viable cells). Significant differences were observed between dissociation combinations as measured by both viability and cell counts (two-way analysis of variance (ANOVA) with Bonferroni post-test, ${ }^{*} P<0.05$, ${ }^{* *} P<0.01$ and ${ }^{* * *} P<0.001$ ). (b) Following selection of the NEDB + enzymes treatment, the effects of different Ficoll preparations were assessed. Dual-density (DD) and commonly used low-density (LD) Ficoll conditions showed statistical differences in cell counts (two-way ANOVA with Bonferroni post-test, ${ }^{*} P<0.05$, NS, not significant). HD, high density. 
Antony, France) or cisplatin (Merck, Darmstadt, Germany) was added at the indicated concentrations. After a $72 \mathrm{~h}$ incubation in the presence or absence of either compound, viability was assessed by WST- 1 and the $50 \%$ inhibitory concentration $\left(\mathrm{IC}_{50}\right)$ was calculated from the corresponding sigmoid analytical curve.

\section{RBD Generation and Production}

H2RBD.mFc, KoRBD.mFc, RD114RBD.mFc and ARBD.rFc immunoadhesins were derived from the sequences encoding the first 178, 253, 222 and 244 amino acids of the human T-cell leukemia virus (HTLV)-2, Koala endogenous retrovirus (KoRV), RD114 and Amphotropic-MLV Env, respectively. The corresponding RBD-encoding sequence was fused to either mouse $(\mathrm{mFc})$ or rabbit $(\mathrm{rFc})$ IgG Fc fragments. All constructs were inserted into the eukaryotic pCSI expression vector $^{24}$ and were transfected into $293 \mathrm{~T}$ cells by a calciumphosphate precipitation method. Cells were washed $16 \mathrm{~h}$ later and incubated for another $48 \mathrm{~h}$ in serum-free Optipro SFM medium (Invitrogen) supplemented with glutamine and non-essential amino acids. Conditioned media were harvested, filtered through $0.45 \mu \mathrm{m}$ pore-diameter filters and concentrated 100 -fold by centrifugation at 3600 r.p.m. on $9 \mathrm{kDa}$ cutoff Icon concentrators (Thermoscientific Pierce, Rockford, IL, USA). Samples were aliquoted and stored at $-80^{\circ} \mathrm{C}$. Each preparation was verified for integrity by western blotting and immunoadhesin concentrations were measured by ELISA using anti-rabbit or anti-mouse IgG Fc.

\section{Cell Analysis by Flow Cytometry}

Cells were stained as follows: $3-5 \times 10^{5}$ viable cells were stained in $\mathrm{PBS} / 2 \%$ FCS with a rat anti-mouse Pan $\mathrm{H}-2$ (CMH-I) mAb coupled to PE (BioLegend, San Diego, CA, USA), a mouse anti-human EpCAM (CD326) mAb coupled to PerCpCy5.5 (BioLegend) and a mouse anti-human CD44 $\mathrm{mAb}$ APC-H7 (BD Biosciences) for $20 \mathrm{~min}$ at $4{ }^{\circ} \mathrm{C}$ in the dark. Cells were washed two times with $\mathrm{PBS} / 2 \%$ BSA and $2 \mathrm{ng} / \mathrm{ml} \mathrm{4}$ 4 $^{\prime} 6^{\prime}$-diamidino-2-phenylindole (DAPI) (Invitrogen) was added. In some cases, cells were fixed in $0.05 \%$ paraformaldehyde after staining.

For detection of apoptotic cells, $3-5 \times 10^{5}$ viable cells were stained in $1 \times$ FLICA (fluorescent ligand inhibitor of caspase-activated) solution using FAM-DEVD-FMK, a specific ligand of active caspase- 3 and -7 (AbD Serotec, Raleigh, NC, USA), according to the manufacturer's recommendations. Following incubation for $1 \mathrm{~h}$ at $37^{\circ} \mathrm{C}$ under $5 \% \mathrm{CO}_{2}$ atmosphere, cells were washed two times with $\mathrm{PBS} / 2 \% \mathrm{BSA}$ and $2 \mathrm{ng} / \mathrm{ml}$ DAPI (Invitrogen) was added.

For apoptosis induction, $\mathrm{HBCx}-14$ was dissociated according to the protocol described above, seeded in 6-well plastic dishes at $1 \times 10^{6}$ cells per well in $2 \mathrm{ml}$ of culture medium supplemented with $10 \%$ FCS and adapted to ex vivo conditions for $72 \mathrm{~h}$. CPT11 (camptothecin) was added, while renewing medium at different times and concentrations. After $48 \mathrm{~h}$, each well was harvested using trypsin. Single-cell suspensions were stained with FLICA following the manufacturer's instructions.

For metabolite transporter labeling, $3-5 \times 10^{5}$ viable cells were incubated in an RBD premix consisting of $3 \mu \mathrm{l}$ of RBD fused to either mouse or rabbit Fc in a total of $50 \mu$ l of medium for $30 \mathrm{~min}$ at $37^{\circ} \mathrm{C}$. Cells were washed once and then incubated with either a secondary AF488-coupled goat anti-mouse antibody (Cell Signaling Technology, Danvers, MA, USA) or a AF647-coupled goat anti-rabbit antibody (Cell Signaling Technology) for $30 \mathrm{~min}$ at $4{ }^{\circ} \mathrm{C}$. Cells were washed once with $\mathrm{PBS} / 5 \% \mathrm{BSA}$ and then subsequently stained with PE-coupled rat anti-mouse Pan H-2 PE (BioLegend) and PerCpCy5.5-coupled mouse anti-human EpCAM (CD326) (BioLegend) mAbs. Cells were washed two times with $\mathrm{PBS} / 5 \% \mathrm{BSA}$ and $2 \mathrm{ng} / \mathrm{ml}$ DAPI was added before flow cytometry analysis.

\section{Flow Cytometry Data Acquisition and Processing}

Cells were analyzed on a standard LSRII (BD Biosciences) with 488, 633 and $407 \mathrm{~nm}$ excitation. 'Fluorescence minus one' (FMO) controls were used to establish negative cutoffs. ${ }^{25}$ Results were calculated in terms of molecule of equivalent soluble fluorochrome (MESF) values as compared with a microsphere template (AF647 and AF488 MESF quantum beads; Bangs Laboratories, Fishers, IN, USA).

$\mathrm{RBD}$ background levels were determined on the basis of the fluorescence detected following staining with the secondary conjugated antibody alone.

Data analyses were performed using the Flowjo software (Tree Star, Ashland, OR, USA).

\section{Statistical Analyses}

The non-parametric Kruskal-Wallis one-way analysis of variance test was used for intergroup comparisons for each transporter expression level. The non-parametric Spearman's rank order measured correlation between the two different methods used to quantify CD44 and CD24. Non-parametric unpaired Mann-Whitney $t$-test underlines the difference in the level of CD44 expression level measured in cytometry versus appreciated by IHC reader. Tukey's method was used as dispersion indication of live cells recovery yields from dissociated tumors, identifying data set outliers outside of 1.5 -fold the interquartile range.

\section{Immunohistochemistry}

Samples were fixed in formalin $(10 \%(\mathrm{vol} / \mathrm{vol})$ in PBS), dehydrated and paraffin embedded. Sections $(4 \mu \mathrm{m})$ were stained with hematoxylin-eosin-safranin according to standard histological procedures in a Leica ST 5020 multistrainer.

For CD44 staining, $4-\mu \mathrm{m}$ sections were adhered to Superfrost Plus slides (Fisher Scientific, Pittsburgh, PA, USA), dried overnight at $40^{\circ} \mathrm{C}$ and heated to $60^{\circ} \mathrm{C}$ for $1 \mathrm{~h}$. Sections were deparaffinized overnight, rehydrated and unmasked in citrate buffer $(10 \mathrm{mM}), \mathrm{pH} 6$, for $20 \mathrm{~min}$ in a $350 \mathrm{~W}$ microwave. The staining was performed in a Nexes 
automat (Ventana Medical Systems; Roche Diagnostics) using a rabbit anti-CD44 (Sigma HPA005785; 1:200 dilution) polyclonal antibody for $30 \mathrm{~min}$ at $37^{\circ} \mathrm{C}$, followed by an avidin-biotin peroxidase complex, and developed by DAB . Slides were counterstained with hematoxylin. Images were captured using a fully motorized microscope (Zeiss Axio Imager Z1; Carl Zeiss, Jena, Germany) with a high-resolution color digital camera (Zeiss HRc) and AxioVision 4.6.3 SP1 software.

\section{Gene Expression Analysis}

The concentration and integrity/purity of each RNA sample were measured using RNA 6000 LabChip kit (Agilent) and the Agilent 2100 bioanalyzer. The DNA microarrays used in this study were the Human Genome U133 Plus 2.0 array containing 54675 probe sets (Affymetrix, Santa Clara, CA, USA). In all, 100 ng of total RNA were amplified and labeled according to the Affymetrix $3^{\prime}$ IVT express protocol. Each batch of targets included an MAQC A sample to control for target preparation and hybridization. Targets were validated according to yield and size of RNA, usually obtained at the Institut Curie molecular biology facility. Targets were hybridized on human and mouse microarrays. Chips were washed and stained on a fluidic station according to the manufacturer's protocol and were scanned using an Affymetrix GCS3000 scanner. Microarray quality control assessment was performed using the R AffyPLM and SimpleAffy packages available from the Bioconductor website. Relative log expression, normalized unscaled standard errors, scaling factor, percentage of 'present' calls, $3^{\prime} / 5^{\prime}$ ratio and average background tests were applied to determine the quality of each experiment. Chip pseudoimages were produced to assess artifacts on arrays that failed to pass the previous quality control tests. Selected arrays were normalized according to the GC-RMA normalization procedure. ${ }^{26}$ Raw data can be obtained from the Institut Curie Microarray Database (http:// microarrays.curie.fr/publications/recherche_translationnelle/ characterization_of_xenografts/files/AffymetrixData.zip).

Statistical analysis, quality control analysis, differential expression analysis and gene ontology analysis were performed by Genosplice Technology (Paris, France).

\section{RESULTS}

Four dissociation protocols, using two types of enzymatic digestions (collagenase III + DNase I with or without trypsin), alone or in combination with NEDB, were tested. Cell recovery obtained with these protocols was assessed in xenografts derived from four different breast cancer models (Figure 1). The addition of the NEDB step before collagenase + DNase treatment generally improved cell yields by an over twofold increase as compared with enzyme treatment alone (Figure 1a). The addition of trypsin to the collagenase/DNase I cocktail had variable and minor effects on cell yields and viability, probably due, at least in part, to tryptic digestion of dead cells. Dissociation with NEDB followed by collagenase III/DNase I (protocol NEDB + E) reproducibly gave the best results, as assessed by total yields of viable cells per gram of tissue. An average of $36 \times 10^{6}$ viable cells per gram of tumor was obtained with protocol $\mathrm{NEDB}+\mathrm{E}$, albeit with some differences between individual xenograft models.

The latter protocol was therefore applied to evaluate the subsequent Ficoll purification steps. Three Ficoll density gradients were tested for the elimination of red blood cells and debris: high $(1.119 \mathrm{~g} / \mathrm{ml})$, low $(1.077 \mathrm{~g} / \mathrm{ml})$ and dual $(1.077+1.119 \mathrm{~g} / \mathrm{ml})$ densities. Fractions were pooled and viable cells counted. The total yields of viable cells were minimally, but repeatedly, higher when using the dual-density Ficoll gradient (Figure 1b). Debris and red blood cell elimination was improved with the $1.119 \mathrm{~g} / \mathrm{ml}$ single-density and dual-density gradients as compared with the low-density gradient.

NEDB incubation followed by collagenase III/DNase I dissociation and subsequent Ficoll gradient purification yielded the highest numbers of good quality viable cells, with a minimum of debris, red cells and apoptotic cells, and was therefore used for subsequent experiments.

\section{Reproducibility of the Final Protocol}

The reproducibility of the aforementioned protocol was tested on eight additional tumor models. The average yield, calculated from 71 different xenografts derived from these eight tumors, ranged from $1.5 \times 10^{6}$ to $1.5 \times 10^{7}$ viable cells per gram of tumor (Figure 2). Although cell yields varied from one model to another, they were impressively reproducible within a given model. No clear explanation could be deduced for this variability, neither stroma infiltration, which could alter dissociation efficiency, nor the number of fibroblasts (data not shown). We did though note that a minimum

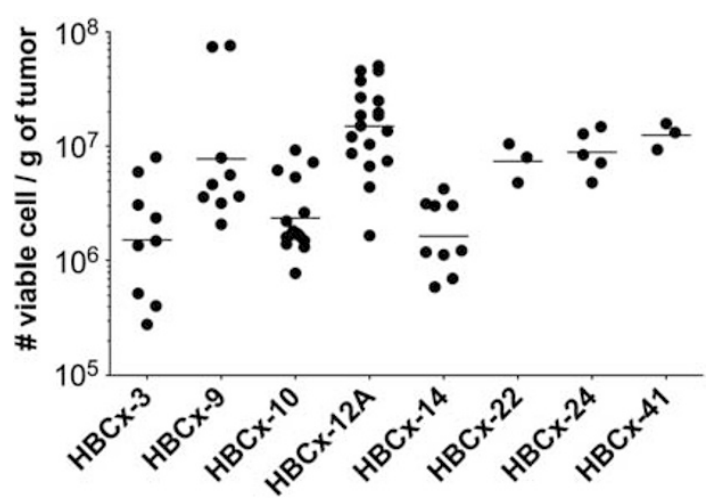

Figure 2 Reproducibility of the optimized protocol for the recovery of viable tumor cells in different xenograft models. Statistical analysis of population dispersion in each data set performed by Tukey's method showed no outlier (all points were within 1.5-fold interquartile range). The yields and viability of isolated tumor cells were determined for eight different human breast cancer tumors removed from a total of 71 xenografts. Each dot represents the value of an independent xenograft and drawbars represent the mean of xenografts from the same tumor model. 
tumor size was required to perform these studies as very small tumors, $<600 \mathrm{~mm}^{3}$, did not yield enough cells to proceed with subsequent analyses. Nonetheless, these results validated the selected protocol and underlined the marked heterogeneity of yields among tumors of different origins.

\section{Flow cytometry Analyses of Dissociated Tumor Cells}

Our gating strategy included a preliminary exclusion of cell debris, aggregates and dead cells (Figure 3). Dead cells were excluded based on DAPI positivity. The vast majority of live cells were not apoptotic as $<15 \%$ of DAPI-negative cells expressed activated caspases-3/7 (as assessed using the fluorescent FAM-DEVD-FMK probe) (Supplementary Data Figure 1). To ensure that caspases were not lost or altered by the dissociation protocol, we tested the induction of apoptosis triggered by camptothecin on cells freshly obtained from the HBCx-14 model (Supplementary Figure 1). Apoptosis is clearly induced by this drug, as revealed by caspase labeling, hence demonstrating that caspases are not lost and remain activable. Concomitant staining with an anti-human EpCAM (CD326) and an anti-mouse pan-H2 (MHC class I) $\mathrm{mAb}$ allowed viable human tumor cells to be discriminated from surrounding mouse stroma and hematopoietic cells (Figure 3e). Single live human cells could then be analyzed, and the surface expression of metabolite transporters determined by flow cytometry (Figure 3f).

The robustness of the dissociated tumor cells was further assessed in ex vivo cultures as attached monolayers. Hence, dissociated tumor cells were cultured to validate their ex vivo viability and the possibility to perform further cytotoxic assays using chemotherapy dose-response experiments. During the 13 days of ensuing culture, the cells showed longterm viability as determined by a WST assay (Figure 4). IC 50 of docetaxel and cisplatin obtained on these cells were well correlated with known in vitro and in vivo data; $\mathrm{HBCX}-41$ is resistant to docetaxel in vivo, and $\mathrm{IC}_{50}$ determined by dose-response curves ex vivo (approximately $40 \mu \mathrm{M}$; Figure $4 \mathrm{~b}$ ) is higher or comparable to $\mathrm{IC}_{50}$ of a set of breast cancer cell lines (Figure 4c). HBCx-41 is resistant to cisplatin in vivo, and $\mathrm{IC}_{50}$ determined by dose-response curves ex vivo (approximately $60 \mu \mathrm{M}$; Figure $4 \mathrm{~b}$ ) is much higher than $\mathrm{IC}_{50}$ of a set of breast cancer cell lines (Figure 4c). Altogether, these data show that tumor cell sensitivity to treatment was concordant to tumor xenograft responsiveness in vivo.

\section{Dissociated Tumor Cells Show the Same Pattern of CD44 Staining as the Tumor Xenograft}

We next assessed whether the dissociated cells were representative of the tumor xenograft and whether cell surface marker integrity was maintained. To this end, we compared CD44 expression, a surface marker that serves as a receptor for hyaluronic acid and is involved in cell-cell interactions, cell adhesion and migration, and that expression was extensively studied in human breast cancers. ${ }^{27} \mathrm{CD} 44$ expression was monitored on sections and dissociated cells from the same tumor by immunohistochemistry and flow cytometry, respectively. Analyses of CD44 staining on a panel of 12
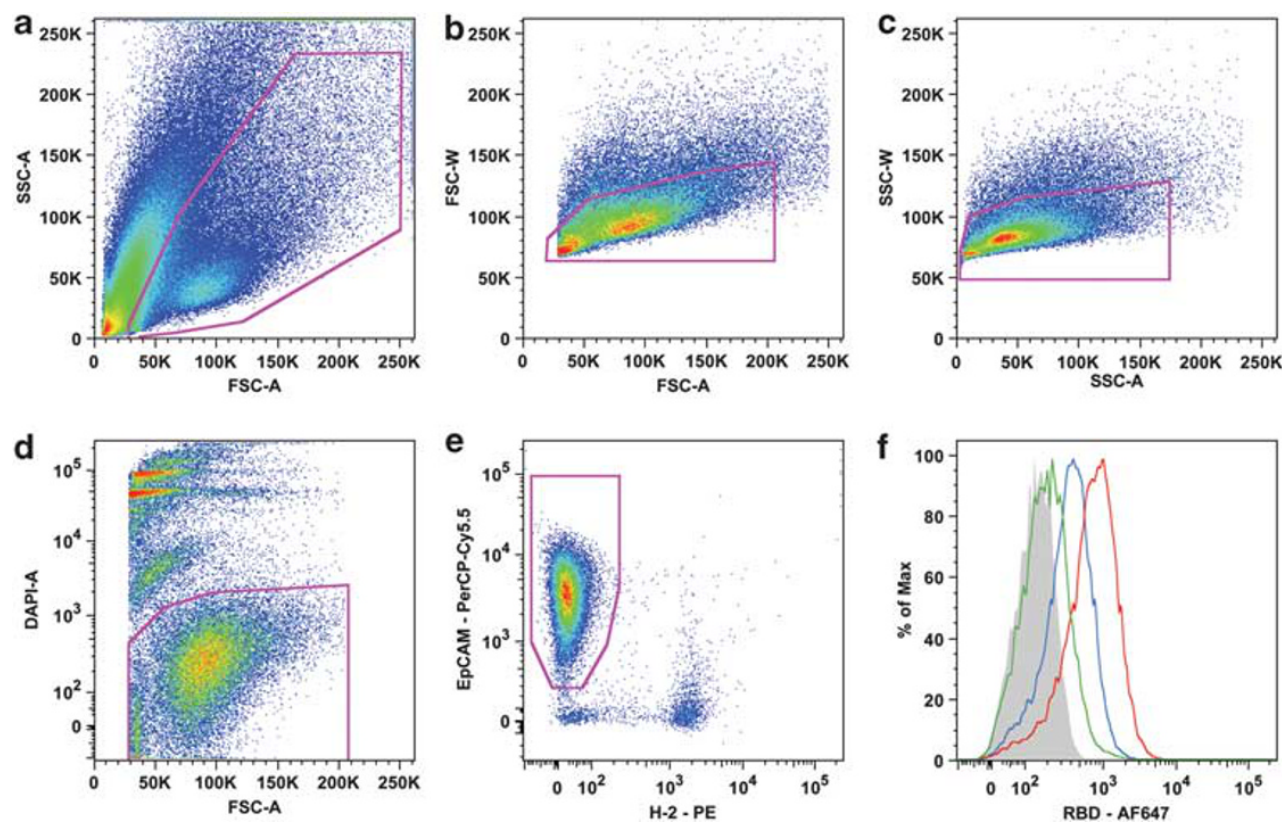

Figure 3 Gating strategy for the analyses and sorting of isolated tumor cells from mice xenografts. (a) Cells are selected on the basis of forward (FSC) and side scatter (SSC) (gated cell population). Aggregates and doublets are excluded using forward (b) and side (c) scatter areas versus their respective width parameters, and (d) dead cells are excluded by 4',6'-diamidino-2-phenylindole (DAPI) staining. (e) Gating of mouse H2-positive and human epithelial cell adhesion molecule (EpCAM)-positive H2-negative tumor cells. (f) The cell surface expression of metabolite transporters on live cells is determined with receptor binding domain (RBD) labeling (green: ASCT2; blue: PiT1; red: PiT2). PerCP-Cy5.5, peridinin chlorophyll protein. 

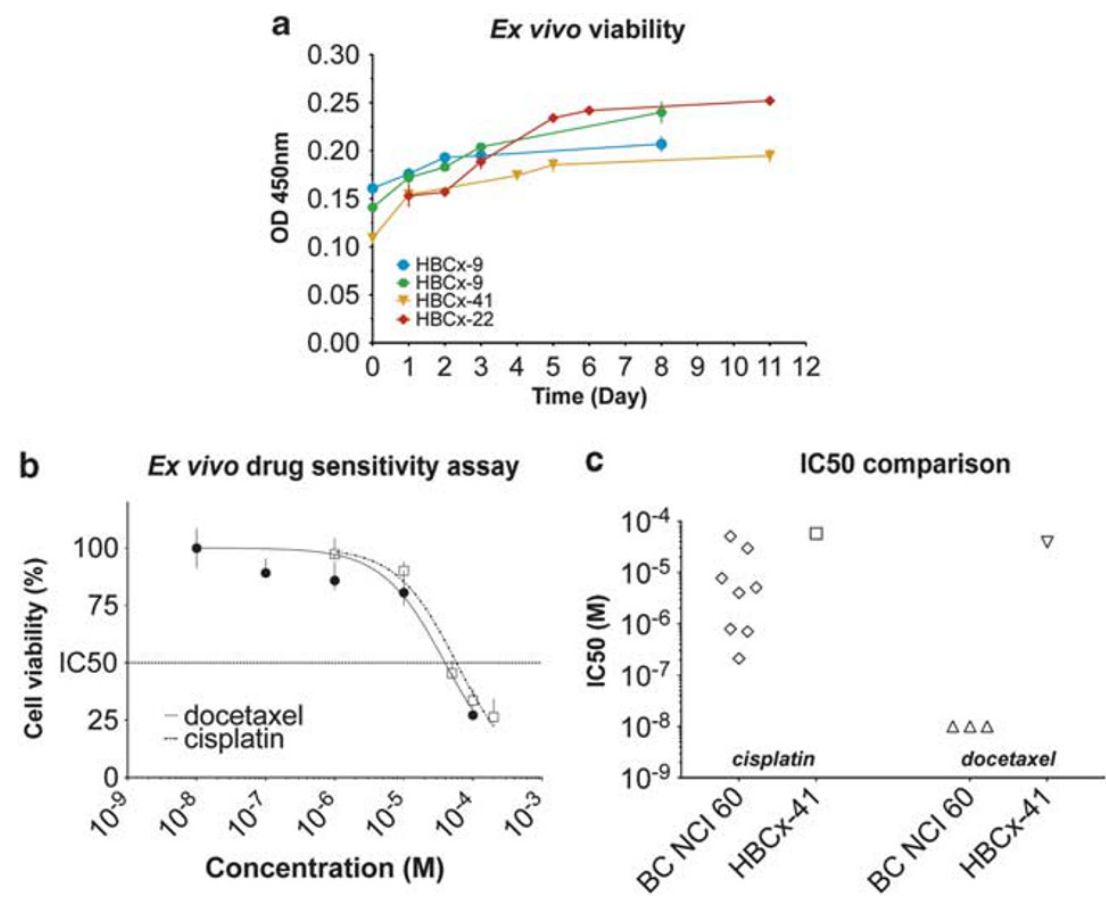

Figure 4 Viability and ex vivo culture of dissociated tumor cells from mouse xenografts. (a) The proliferation of dissociated cells was analyzed by a WST assay over a 13-day period. (b) Dissociated HBCx-41 cells show a dose-dependent toxicity to docetaxel and cisplatin during ex vivo culture. Treatments were continued for 3 days in dissociated cells that were previously cultured for 3 days. (c) The $50 \%$ inhibitory concentration (IC 50 ) of docetaxel and cisplatin ex vivo on $\mathrm{HBCx}-41$-isolated cells (square and downward triangle) is compared with $\mathrm{IC}_{50}$ of the two drugs determined by the National Cancer Institute ( $\mathrm{NCl}$ ) on a set of human breast cancer cell lines (diamonds and upward triangle) (http://dtp.cancer.gov/dtpstandard/ cancerscreeningdata/meangraphHelpFile.jsp). OD, optical density.

different human breast cancer xenografts showed that the proportions and labeling intensities of CD44 + cells by flow cytometry were concordant with the IHC staining (Figure 5). As shown for two representative tumors with different levels of CD44 expression (Figures 5a and b), the selected protocol does not appear to affect either the percentages or staining intensity of the CD44 marker. Moreover, this concordance was observed on the ensemble of xenografts that were compared using the two techniques and was confirmed by statistical analysis. A statistical difference exists between tumors classified as medium and high CD44 expression in IHC, relative to their MFI determined by cytometry (Figure 5c), and a correlation between positive cells for CD44 in IHC compared with cytometry is characterized by parameters of $r=0.65$, with a $P$-value of 0.02 , demonstrating a good correlation between these data set (Figure $5 \mathrm{~d}$ ).

Furthermore, we showed that the expression level or mRNA and the percentage of labeled cells were correlated for both CD44 and CD24, another commonly used surface marker in breast cancer phenotyping, supporting evidence for the maintenance of antigenicity integrity (Figures $5 e$ and $\mathrm{f}$ ).

\section{Detection of Metabolite Transporters and Quantification of their Cell Surface Expression}

There is a paucity of antibodies allowing a reliable detection of nutrient and metabolite transporters at the cell surface. ${ }^{5,6}$ Here, cell surface expression of four metabolite transporters was monitored using retroviral RBDs that bind to the exofacial determinants of Glut1, ASCT2, PiT1 and PiT2. These transporters are cognate receptors for HTLV, ${ }^{11,28}$ feline RD114 endogenous retrovirus, ${ }^{29} \mathrm{KoRV}^{30}$ and amphotropic murine leukemia virus ${ }^{7,8,30,31}$ Env, respectively. We assessed cell surface expression of these transporters in five human breast cancer models with different molecular characteristics (Supplementary Table 1) and found that the transporter expression profile of each tumor was unique (Figure 6).

Using this new multiplex assay, we observed that tumors do not uniformly express high or low levels of nutrient transporters, but rather have distinctive patterns that were remarkably consistent between xenografts that originated from the same tumor. For example, cell surface expression of ASCT2 was highly variable between models $(P<0.01)$, with HBCx-3 cells exhibiting 50-fold higher levels than those detected on $\mathrm{HBCX}-4 \mathrm{~A}$ cells. Notably though, high expression of one transporter was not necessarily associated with high levels of other transporters; the high ASCT2 expression level detected on $\mathrm{HBCx}-3$ cells was associated with low levels of both PiT1 and PiT2 inorganic phosphate transporters.

Glut1 levels in HBCX-4A and HBCX-24 cells were 50\% lower than those detected in the other three models $(P<0.02)$. Furthermore, PiT1 and ASCT2 levels were significantly reduced in $\mathrm{HBCx}-4 \mathrm{~A}$ cells as compared with the four other models $(P<0.01)$, while the second phosphate transporter, PiT2, was expressed at levels comparable to those 
a HBCx-3 CD44 IHC score low
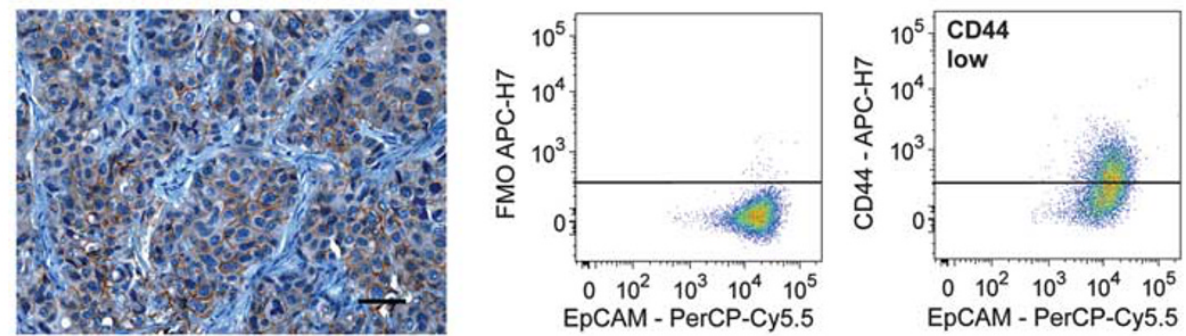

b HBCX-4A CD44 IHC score high
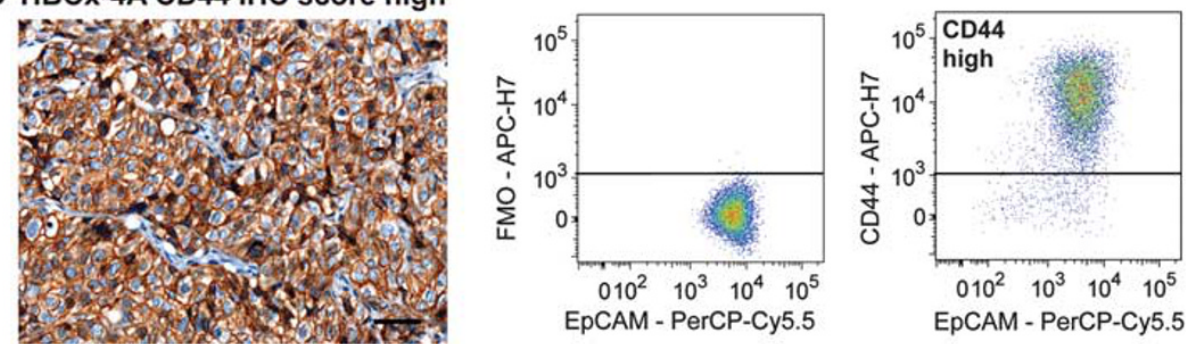

C

CD44 staining intensity

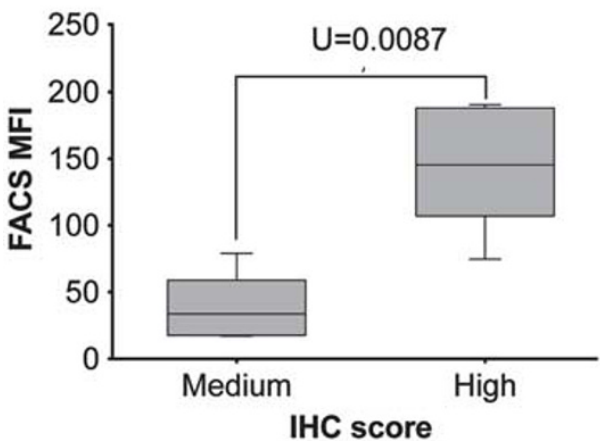

d CD44 positive cell number

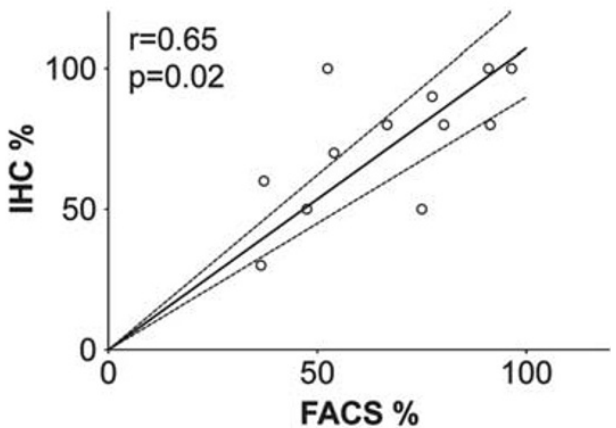

e

CD44 expression

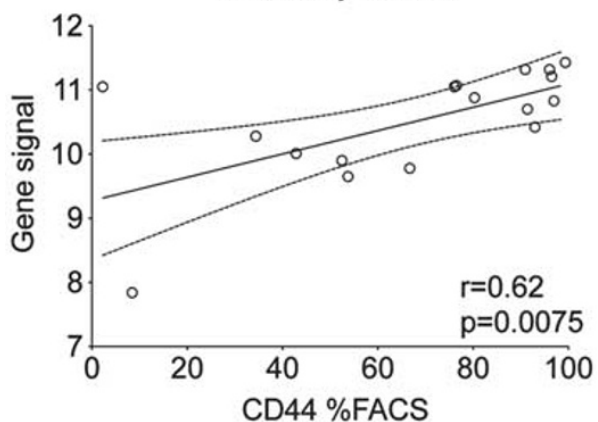

f

CD24 expression

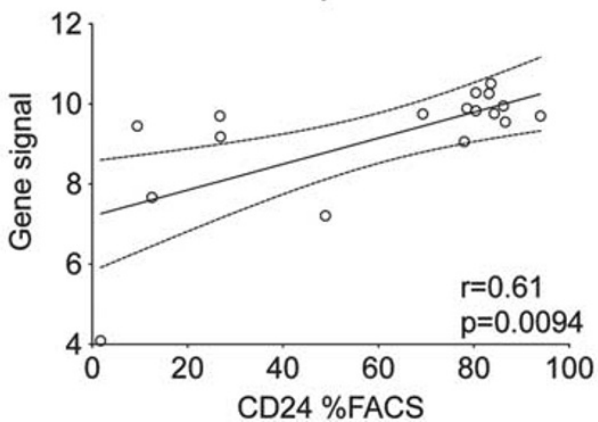

Figure 5 The level of CD44 expression detected on dissociated tumor cell xenografts by flow cytometry closely correlates with tumor block immunohistochemistry data. The level of CD44 in breast cancer xenografts was determined by immunohistochemistry and representative (a) low (HBCx-3)- and (b) high (HBCx-4A)-expressing tumors are shown (scale bars $20 \mu \mathrm{m})$. The corresponding level of CD44 in dissociated epithelial cell adhesion molecule (EPCAM)-positive tumor cells was monitored by flow cytometry. FMO (fluorescence minus one) control staining is shown in the left panels and CD44 expression in the right panels. (c) Comparison of CD44 expression, monitored by flow cytometry on dissociated cells and immunohistochemistry (IHC) performed on tumor sections of 11 breast cancer xenografts and classified as medium and high. Non-parametric unpaired Mann-Whitney $t$-test shows reliable difference between the two groups. (d) Linear regression curve, Spearman's rank-order correlation coefficient $r$ and two-tailed $P$-value with $95 \%$ confidence interval of CD44 expression on dissociated cells by flow cytometry versus IHC on tumor sections. (e) Correlation between CD44 mRNA determined by Affymetrix chip and percentage of positive cells for CD44 immunostaining by flow cytometry, linear regression curve, Spearman's $r$ and two-tailed $P$-value at 95\% confidence interval. (f) Correlation between CD24 mRNA determined by Affymetrix chip and percentage of positive cells for CD24 immunostaining by flow cytometry, linear regression curve, Spearman's $r$ and two-tailed $P$-value with a 95\% confidence interval. APC, allophycocyanin; FACS, fluorescence-activated cell sorter; MFI, mean fluorescence intensity; PerCP-Cy5.5, peridinin chlorophyll protein. 


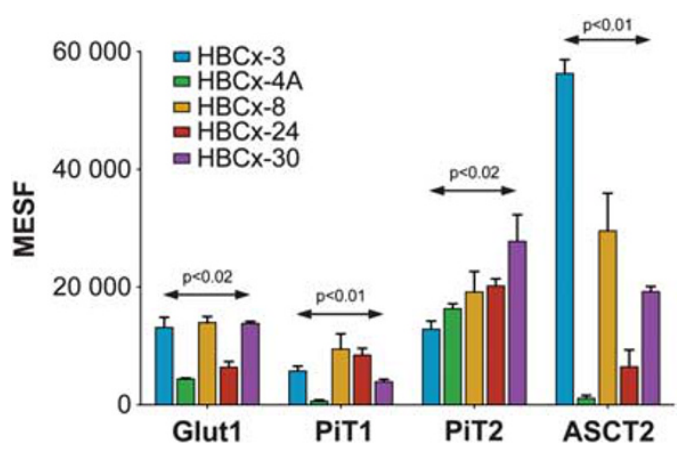

Figure 6 Surface expression of nutrient transporters on five breast cancer xenograft models. The expression of each transporter is plotted as a function of molecule of equivalent soluble fluorochrome (MESF). Results are expressed as the means \pm s.d. of independent xenografts of each tumor (HBCx-3, $n=6$; HBCx-4A, $n=3$; HBCx-8, $n=3 ; \mathrm{HBCx}-24, n=5$; and $\mathrm{HBCx}-30, n=3)$. $P$-values were calculated using a non-parametric analysis of variance (ANOVA) Kruskal-Wallis test, meaning that, considering one receptor binding domain (RBD) expression, the five studied models are statistically different from each other.

found in other tumors. Even though PiT2 was detected on all tumors, its expression on $\mathrm{HBCx}-30$ cells was significantly higher than that detected on $\mathrm{HBCx}-3$ cells $(P<0.02)$. Thus, these data point to a high variability in metabolite transporter expression in breast cancer tumors.

The variability in the cell surface transportome of human tumors was revealed by our strategy, combining a dedicated dissociation protocol with a novel cell surface detection method for metabolite transporters. This method will allow the specific metabolite profiles of individual human breast cancers to be elucidated.

\section{DISCUSSION}

Dissociation of tumor samples to produce high yields of viable cells with intact antigens and function is a challenging task. The dissociation protocol developed in this study allows good recovery of viable cells from epithelial human breast cancer xenografts in mice, sufficient for phenotypic analyses by flow cytometry and ex vivo culture.

Short preincubation times with NEDB composed of chelators minimized enzymatic overdigestion of tissue and cell membrane, preserving cell viability and resulting in satisfactory cell yields. The subsequent enzymatic digestion using collagenase III and DNAse I, gave high yields of viable cells with intact surface marker expression. Removal of dissociated cells into cold medium supplemented with 30\% FCS, between each digestion step, was important for maintaining cell integrity. The dual gradient Ficoll purification allowed the cells of interest to be separated from both debris and red cells. This combined dissociation and purification protocol produced a higher quality cell preparation than the enzymatic digestion and ammonium chloride red cell lysis steps commonly reported in the literature for the dissociation of tumoral or normal mammary and other tissues. ${ }^{17,20}$ This optimized protocol produced high-quality cellular material in $<3 \mathrm{~h}$, and allowed subsequent cell analyses to be performed on the same day.

While the yield of viable cells varied significantly between tumor models, it remained reproducible for xenografts that originated from the same patient, underscoring the reliability of the protocol and the intrinsic differences between the different models. Although we found that these differences did not correlate with the level of stromal infiltration, other disparities, including stromal architecture, vasculature, fibrosis, fat composition or degrees of necrosis, which are commonly observed in xenografts, may influence the distinctive yield efficiency of each model. Also, we did not observe any relationship between the tumor phenotypes and dissociation efficiency.

The dissociation procedure requires amounts of cells that would necessitate more materials than what is usually directly obtained from the smallest tumors that are removed from patients. In this context, our procedure seems to be applicable, thus far, to the largest tumors, such as some of those obtained after surgery. Nevertheless, as the xenografted tumor models selected here mirror faithfully the molecular and phenotypic profiles of parental tumors, ex vivo signatures of xenografts to anticancer drug responses could still be established with our method. Although the delay between the first tumor transplantation into mice and the obtention of a corresponding xenograft will extend over many months in most cases, this experimental opportunity remains of high interest in the view of therapeutic combinations of targeted compounds. Thus, in the case of pancreatic carcinoma of particularly pejorative prognosis, it has been demonstrated that preclinical data obtained in xenografts could be transferred into therapeutic choice for the corresponding patients. $^{32}$ Improvement in the sensitivity of our procedure will hopefully help adapting it to primary tumors.

Cell surface markers can be easily destroyed during enzymatic treatment. To verify that the cell populations that were produced upon dissociation were representative of the original tumor and that markers of interest were quantifiable, CD44, an integral cell surface tumor marker, was analyzed. A flow cytometric analysis of CD44 expression on the dissociated cells showed a high correlation with the staining intensity on the original xenograft, which was assessed by IHC, and with mRNA level as determined by transcriptomic analysis (also verified for CD24). Although this does not ensure that all antigens of interest are intact upon our dissociation protocol, it strongly argues that the single dissociated cells are representative of the tumor population and points to the integrity of specific and structurally distinct surface antigens. Accordingly, the dissociated cell preparations were easily distinguished following staining with human anti-EpCAM and mouse anti-H2 mAbs. Furthermore, it appears that the multimembrane-spanning transporter proteins assessed here are not damaged by the dissociation treatment. Indeed, these transporters, all of which are multimembrane spanning molecules, which serve as receptors for 
retroviruses, remained accessible to their cognate Env following collagenase or trypsin treatment.

The four nutrient transporters that we monitored for this study are relevant at different levels to cell metabolism related to tumorigenesis. Thus, Glut1 and ASCT2 are key to cell proliferation and oncogenic processes; this has been an ongoing research field for Glut1 (or SLC2A1) since Warburg's genial work in the $1950 \mathrm{~s}^{4}$ and ASCT2 (or SLC1A5) is a transporter of neutral amino acids regulating proliferation versus autophagy associated to mTOR pathways and corresponding mTOR complexes. ${ }^{33}$ PiT1 and PiT2 are inorganic phosphate transporters ${ }^{31}$ and, interestingly, PiT1 has been shown to regulate cell proliferation independently of phosphate transport. ${ }^{34}$ Moreover, nutrient transporters are generally thought to transport multiple metabolites and Glut1, for example, has been shown to be a major transporter of dehydroascorbic acid in certain cellular contexts. ${ }^{12}$

Therefore, one of the originalities of the present work lies in the surface detection on live cells of those transporters that are receptors to gammaretrovirus Env. Although other transporters, such as Glut isoforms, transporters of folate, glutamate, organic cations or platinum and many others, would also be relevant and valuable to monitor, their detection at the cell surface has been nearly impossible to perform on live cells due to the notorious lack of reliable antibodies directed against exofacial epitopes of multipass nutrient transporters. ${ }^{5,6}$ Here, the use of four ligands that bind Glut1, ASCT2, PiT1 and PiT2 did not allow a predictive distinction of the tumor phenotype. However, we are exploring a larger panel of tumors with these ligands and a series of additional ligands derived from a variety of gammaretroviruses whose Env recognizes a receptor on human cells. ${ }^{7,8}$ We trust that a more thorough exploration may provide distinctive tumor signatures.

A growing body of literature points at the pleiotropic roles of nutrient transporters in cell metabolism and physiol$\mathrm{ogy}^{35,36}$ and situate them as prognostic markers in certain cancers. $^{37,38}$ Thus, tools, such as those described here, are urgently needed to quantify cell surface expression of these transporters. The identification of transporter profiles will help us to elucidate the metabolism of individual tumors, and will refine diagnostic, prognostic and therapeutic response assessments as well as foster drug discovery and development.

Supplementary Information accompanies the paper on the Laboratory Investigation website (http://www.laboratoryinvestigation.org)

\section{ACKNOWLEDGEMENTS}

We thank Elisabetta Marangoni, Ludmilla De Plater and Franck Assayag (Laboratory of Preclinical Investigation, Institut Curie) for providing breast cancer xenografts; Kelly Lundsten (BioLegend) for her support in multicolor flow cytometry; Aurélie DuThanh, Donatella Giovannini, Cedric Mongellaz and Madakasira Lavanya for kindly sharing time and discussions on this project; Joachim Denner (RKI, Berlin) and François-Loïc Cosset (ENS, Lyon) for sharing KoRV and RD114 env plasmids, respectively. We also thank Genosplice, Pierre De La Grange and Frédéric Lemoine for their advise and process in transcriptomic field, and Pr Bellet for accommodating some experiments in his lab. Expertise of the MRI-RIO imaging and the production of recombinant proteins platforms (in Montpellier) are acknowledged. This work was supported, in part, with grants from INCa/DGOS program (Translational research), and from ANR Glutstem, AFM, FRM, ARC and the European Community (Contract No. LSHC-CT-2005-018914 'ATTACK').

\section{DISCLOSURE/CONFLICT OF INTEREST}

Vincent Petit previously worked as a research engineer at CNRS, and presently holds an executive position (COO) at METAFORA biosystems; he is a shareholder in the company. METAFORA biosystems focuses on metabolite transporters in physiological and pathological conditions. Marc Sitbon is a co-founder of METAFORA biosystems.

1. Hanahan D, Weinberg RA. Hallmarks of cancer: the next generation. Cell 2011;144:646-674.

2. Hsu PP, Sabatini DM. Cancer cell metabolism: Warburg and beyond. Cell 2008;134:703-707.

3. Rubakhin SS, Romanova EV, Nemes $P$, et al. Profiling metabolites and peptides in single cells. Nat Methods 2011;8(Suppl):S20-S29.

4. Ganapathy V, Thangaraju M, Prasad PD. Nutrient transporters in cancer: relevance to Warburg hypothesis and beyond. Pharmacol Ther 2009;121:29-40.

5. Kinet $S$, Swainson L, Lavanya $M$, et al. Isolated receptor binding domains of HTLV-1 and HTLV-2 envelopes bind Glut-1 on activated CD4 + and CD8 + T cells. Retrovirology 2007;4:31.

6. Yonezawa $A$, Masuda $S$, Katsura $T$, et al. Identification and functional characterization of a novel human and rat riboflavin transporter, RFT1. Am J Physiol Cell Physiol 2008;295:C632-C641.

7. Manel N, Battini JL, Taylor N, et al. HTLV-1 tropism and envelope receptor. Oncogene 2005;24:6016-6025.

8. Overbaugh J, Miller AD, Eiden MV. Receptors and entry cofactors for retroviruses include single and multiple transmembrane-spanning proteins as well as newly described glycophosphatidylinositol-anchored and secreted proteins. Microbiol Mol Biol Rev 2001;65:371-389.

9. Lagrue $E, A b e H$, Lavanya $M$, et al. Regional characterization of energy metabolism in the brain of normal and MPTP-intoxicated mice using new markers of glucose and phosphate transport. J Biomed Sci 2010;17:91.

10. Loisel-Meyer S, Swainson L, Craveiro M, et al. Glut1-mediated glucose transport regulates HIV infection. Proc Natl Acad Sci USA 2012;109:2549-2554.

11. Manel N, Kim FJ, Kinet $S$, et al. The ubiquitous glucose transporter GLUT-1 is a receptor for HTLV. Cell 2003;115:449-459.

12. Montel-Hagen $\mathrm{A}$, Kinet $\mathrm{S}$, Manel $\mathrm{N}$, et al. Erythrocyte Glut1 triggers dehydroascorbic acid uptake in mammals unable to synthesize vitamin C. Cell 2008;132:1039-1048.

13. Swainson L, Kinet $\mathrm{S}$, Manel $\mathrm{N}$, et al. Glucose transporter 1 expression identifies a population of cycling CD4 + CD8 + human thymocytes with high CXCR4-induced chemotaxis. Proc Natl Acad Sci USA 2005;102:12867-12872.

14. Decaudin D. Primary human tumor xenografted models ('tumorgrafts') for good management of patients with cancer. Anticancer Drugs 2011;22:827-841.

15. Derose YS, Wang G, Lin YC, et al. Tumor grafts derived from women with breast cancer authentically reflect tumor pathology, growth, metastasis and disease outcomes. Nat Med 2011;17:1514-1520.

16. Garber K. From human to mouse and back: 'tumorgraft' models surge in popularity. J Natl Cancer Inst 2009;101:6-8.

17. Brockhoff G, Fleischmann S, Meier A, et al. Use of a mechanical dissociation device to improve standardization of flow cytometric cytokeratin DNA measurements of colon carcinomas. Cytometry 1999;38:184-191.

18. Corver WE, Cornelisse CJ, Hermans J, et al. Limited loss of nine tumorassociated surface antigenic determinants after tryptic cell dissociation. Cytometry 1995;19:267-272. 
19. Haghighat N, McCandless DW, Geraminegad P. Responses in primary astrocytes and C6-glioma cells to ammonium chloride and dibutyryl cyclic-AMP. Neurochem Res 2000;25:277-284

20. Davidson WF, Parish CR. A procedure for removing red cells and dead cells from lymphoid cell suspensions. J Immunol Methods 1975;7:291-300.

21. Rubenstein $\mathrm{M}$, Anderson KM. Isolation of viable rat ventral prostate epithelial and nonepithelial cells. Endocrinology 1980;106:530-540.

22. Marangoni E, Vincent-Salomon A, Auger N, et al. A new model of patient tumor-derived breast cancer xenografts for preclinical assays. Clin Cancer Res 2007;13:3989-3998.

23. Workman P, Aboagye EO, Balkwill F, et al. Guidelines for the welfare and use of animals in cancer research. Br J Cancer 2010;102 1555-1577.

24. Battini JL, Rasko JE, Miller AD. A human cell-surface receptor for xenotropic and polytropic murine leukemia viruses: possible role in $\mathrm{G}$ protein-coupled signal transduction. Proc Natl Acad Sci USA 1999;96:1385-1390.

25. Roederer M. Compensation in flow cytometry. Curr Protoc Cytom 2002; Chapter 1(Unit 1):14.

26. Irizarry RA, Bolstad BM, Collin F, et al. Summaries of Affymetrix GeneChip probe level data. Nucleic Acids Res 2003;31:e15.

27. Gotte M, Yip GW. Heparanase, hyaluronan, and CD44 in cancers: a breast carcinoma perspective. Cancer Res 2006;66:10233-10237.

28. Manel N, Battini JL, Sitbon M. Human T cell leukemia virus envelope binding and virus entry are mediated by distinct domains of the glucose transporter GLUT1. J Biol Chem 2005;280: 29025-29029.

29. Tailor CS, Nouri A, Zhao Y, et al. A sodium-dependent neutralamino-acid transporter mediates infections of feline and baboon endogenous retroviruses and simian type D retroviruses, J Virol 1999;73:4470-4474.

30. Oliveira NM, Farrell KB, Eiden MV. In vitro characterization of a koala retrovirus. J Virol 2006;80:3104-3107.

31. Kavanaugh MP, Miller DG, Zhang W, et al. Cell-surface receptors for gibbon ape leukemia virus and amphotropic murine retrovirus are inducible sodium-dependent phosphate symporters. Proc Natl Acad Sci USA 1994;91:7071-7075.

32. Hidalgo M, Bruckheimer E, Rajeshkumar NV, et al. A pilot clinical study of treatment guided by personalized tumorgrafts in patients with advanced cancer. Mol Cancer Ther 2011;10:1311-1316.

33. Nicklin $\mathrm{P}$, Bergman $\mathrm{P}$, Zhang $\mathrm{B}$, et al. Bidirectional transport of amino acids regulates mTOR and autophagy. Cell 2009;136:521-534.

34. Beck L, Leroy $C$, Salaun $C$, et al. Identification of a novel function of PiT1 critical for cell proliferation and independent from its phosphate transport activity. J Biol Chem 2009;284:31363-31374.

35. Barnes K, Mclntosh E, Whetton AD, et al. Chronic myeloid leukaemia: an investigation into the role of Bcr-Abl-induced abnormalities in glucose transport regulation. Oncogene 2005;24:3257-3267.

36. Todorova VK, Kaufmann Y, Luo S, et al. Tamoxifen and raloxifene suppress the proliferation of estrogen receptor-negative cells through inhibition of glutamine uptake. Cancer Chemother Pharmacol 2011;67:285-291.

37. Ichinoe M, Mikami T, Yoshida T, et al. High expression of L-type aminoacid transporter 1 (LAT1) in gastric carcinomas: comparison with noncancerous lesions. Pathol Int 2011;61:281-289.

38. Lee $\mathrm{YY}$, Choi $\mathrm{CH}$, Do IG, et al. Prognostic value of the copper transporters, CTR1 and CTR2, in patients with ovarian carcinoma receiving platinum-based chemotherapy. Gynecol Oncol 2011;122: $361-365$. 